\title{
Experimento massa-mola: uma abordagem dinâmica para o ensino das funções
}

Marília Zabel

Ivanete Zuchi Siple

\section{Resumo}

O estudo de funções é considerado um tópico relevante no ensino da matemática estando presente nos diversos níveis de formação, desde o fundamental até o ensino superior. Sua relevância pode ser explicada devida a ampla aplicação deste conceito nas diversas áreas do conhecimento, por meio da análise de fenômenos, descrições de regularidades e interpretações de interdependência e generalizações. Desta forma, este artigo tem como principal objetivo apresentar uma sequência didática para abordar o conceito de função de forma dinâmica. A atividade proposta visa introduzir o conceito de função por meio da compreensão das grandezas envolvidas e a relação entre elas, articulando os diferentes registros de representação, mediados pelo uso de tecnologias. A atividade proposta neste trabalho pode representar um recurso importante tanto para professores do Ensino Básico quanto do Superior, podendo ser adaptadas de acordo com a realidade de cada turma. Na experimentação realizada observamos que esse tipo de abordagem diferenciada pode proporcionar aos alunos um conhecimento mais efetivo e significativo, pois propiciam momentos de discussões, interpretações e compreensão do objeto matemático, bem como pode se tornar um elemento motivador em relação à disciplina ou a determinados conteúdos.

Palavras-chave: Atividades experimentais, Sequência didática, Recursos tecnológicos, Ensino de funções.

\section{Abstract}

The study of functions is considered a relevant topic in mathematics, as it is present in different school levels, from primary up to university education. Its relevance can be explained due to the wide application of this concept in several areas of knowledge through the analysis of phenomena, descriptions of regularities, and interpretations of interdependence and generalizations. Thus, this article has as its main objective to present a sequence of teaching activities activity to address the concept of functions in a dynamic way. The following proposal introduces the concept of functions through an understanding of the quantities involved and the relationship between them in experiments, articulating the different registers of representation, mediated by the use of technologies. The activity proposed in this paper may represent an important resource for teachers of both basic education and higher education, which may be adapted according to the reality of each class. In the experimental work done for this project, it was observed that this kind of differentiated approach can provide students with more effective and meaningful knowledge, since it allows time for discussions, interpretations, and understanding of the mathematical subject, and can become a motivating factor regarding the discipline or certain content.

Key words: Experimental activities, Sequence of teaching activities, Technological resources, Teaching of functions. 


\section{Introdução}

O conceito de função é importante não somente para a matemática como para as ciências em geral, sendo utilizado, por exemplo, como um instrumento necessário para descrever, interpretar e prever fenômenos. Segundo Silva e Rezende (1996), a interpretação do conceito de função como relação entre quantidades variáveis foi, sem dúvida, a mais utilizada pelos matemáticos dos séculos XVIII e XIX, devido ao surgimento da física quantitativa que, para o estudo dos fenômenos naturais, buscou quantificar e estabelecer relações entre as grandezas envolvidas.

Entretanto, no ensino básico ou superior, a ideia mais utilizada para introduzir o conceito de função é a de relação entre conjuntos. Ou seja, de que cada elemento $x$ de um conjunto $A$ se associa um único elemento $f(x)$ de outro conjunto $B$, segundo uma relação definida de $A$ em $B$. Esta interpretação é estática e tem um caráter mais formal que as demais, sendo geralmente dada maior ênfase nas propriedades algébricas da função, bem como na apresentação analítica direta de uma função.

O entendimento das funções dentro de uma estrutura axiomática é tão importante quanto a identificação do seu significado em aplicações, visto como um dos objetivos principais do ensino da matemática, como comprova os Parâmetros Curriculares Nacionais (PCN's):

O estudo das funções permite ao aluno adquirir a linguagem algébrica como a linguagem das ciências, necessária para expressar a relação entre grandezas e modelar situações-problema, construindo modelos descritivos de fenômenos $e$ permitindo várias conexões dentro e fora da própria matemática. Assim, a ênfase do estudo das diferentes funções deve estar no conceito de função e em suas propriedades em relação às operações, na interpretação de seus gráficos e nas aplicações dessas funções. (PCN+, 2002, p.165).

Diante disso, percebemos a necessidade de criar situações dinâmicas para o ensino de matemática, principalmente no que se refere ao conteúdo de funções. Essas podem propiciar a compreensão do conceito de função por meio da identificação das grandezas envolvidas e a relação entre elas, podendo ser vista como uma oportunidade para a formação do aluno, tanto em termos de conteúdo específico, quanto um fator motivador pela disciplina.

Temos como hipótese que é fundamental no contexto do ensino de funções os alunos saberem identificar as grandezas e as relações entre elas, seja numa situação hipotética ou real. É com essa compreensão que os alunos poderão verificar a tendência entre as grandezas, estabelecer a lei de formação matemática que melhor descreve o comportamento dos dados, bem como fazer conjecturas a respeito da situação problema. Outro fator importante para o ensino das funções é a articulação entre os diferentes registros de representação da mesma, fundamentados na teoria de Representação Semiótica (DUVAL, 1993). 
Diante disso, o desenvolvimento de atividades experimentais aliada com os recursos tecnológicos torna-se um elemento motivador para o ensino de funções, pois possibilita criar situações nas quais é possível explorar os diferentes registros de representações a partir de dados obtidos experimentalmente. O presente artigo apresenta uma sequência didática desenvolvida e aplicada para introduzir o conceito de função por meio da utilização de um experimento prático e mediado pelas potencialidades das ferramentas tecológicas, buscando trazer as reflexões sobre a aplicação deste experimento.

\section{Fundamentação teórica}

$\mathrm{Na}$ fundamentação teórica buscamos apresentar um embasamento referente as possibilidades de se trabalhar o conceito de função em atividades experimentais que propiciem a identificação das grandezas envolvidas, bem como a relação entre elas, nos apoiando na articulação dos diversos Registros de Representação (DUVAL, 1993) e nas potencialidades das ferramentas tecnológicas, contextualizando com o surgimento das funções.

Quando se fala em função, podemos representá-la através do seu gráfico, descrevê-la pela lei que a define ou mostrá-la através de pontos em uma tabela.

Essas representações são importantes, pelo fato de proporcionarem diferentes registros de um mesmo objeto matemático. Esses registros, de acordo com Machado (2003), servem para designar os diferentes tipos de representação semiótica utilizados na matemática - sistemas de numeração, figuras geométricas, escritas algébricas e formais, representações gráficas e a linguagem natural - a completa aprendizagem de um determinado conceito matemático supõe a compreensão de, ao menos, dois registros, deste conceito.

Diante disso, Duval (1995 apud REIS, 2011), afirma que a compreensão matemática está ligada à capacidade de mudar de registro, assim, com essa articulação o aluno reconhece o mesmo objeto matemático em diferentes representações. Ou seja, o aluno cria condições para modificar formulações ou representações de informação durante a resolução de um problema. Esse processo é considerado muito importante, como afirma Reis (2011, p. 44), "passar de um registro de representação para o outro possibilita explicar as propriedades ou os aspectos diferentes de um mesmo objeto na compreensão da matemática".

Aliamos o estudo das diferentes formas de representação com as atividades experimentais que possibilitam momento de discussão, interpretação e limitação de resultados obtidos experimentalmente, possibilitando o confronto das diferentes representações de um mesmo objeto.

Pinho (2006) contribui para a definição de Atividade Experimental afirmando que: 
Atividades experimentais são experimentos que propiciam ao estudante observar, medir, verificar, comprovar, descobrir, simular, ilustrar através de um conjunto experimental (equipamentos) situações, fatos, leis, princípios, causas e efeitos...em Ciências. (PINHO, 2006 apud QUEIROZ; RAMOS, 2007, p. 29)

Assim, podemos ver a atividade experimental como uma atividade desenvolvida num ambiente criado para esse fim, envolvendo os alunos em experiências de aprendizagem planejadas, interagindo com materiais para observar e compreender fenômenos, como propõe Neves (2007).

As experimentações são consideradas importantes ferramentas de ensino para o desenvolvimento de uma aprendizagem significativa em ciências, dando a oportunidade para alunos se expressarem e avaliarem suas ideias e modelos científicos.

Quando falamos em atividades experimentais, nos remetemos às disciplinas de física, química e biologia acreditando que só é possível trabalhar com esse tipo de atividade nessas disciplinas. Vários artigos e teses apresentam a matemática como ferramenta para a interpretação dos experimentos, como podemos verificar neste trecho:

“...para resolver estas situações problematizadas conta-se com uma importante ferramenta, ou seja, a Matemática. Desse modo, os modelos matemáticos exercem papel relevante em todo o desenvolvimento da Física, uma vez que compõem uma tríade fundamental para esta área da Ciência (Lozada et al., 2006): a Física, acima de tudo, apoia-se em formulação de teoria, elaboração de um modelo matemático compatível e experimentação, ..." (MORRONE et al., 2006, p. 2).

Porém, para ensinarmos matemática podemos também utilizar metodologias que envolvam experimentos e, a partir deles, formalizar os conceitos matemáticos. A utilização dos experimentos em sala de aula também permite uma abordagem mais dinâmica e envolvente dos conteúdos. A atividade prática contribui para dar um sentido real do objeto a ser estudado. Assim, os experimentos realizados, podem facilitar o entendimento dos alunos do determinado conteúdo, bem como estimular seu aprendizado.

Enfim, a partir de experimentações vamos obter alguns dados e estes podem possibilitar a criação de conjecturas e a descoberta de resultados matemáticos desconhecidos e/ou frágeis.

É interessante aliar a utilização dos recursos tecnológicos à experimentação, pois o trabalho experimental é algo dinâmico, onde exploramos os vários tipos de representações simultaneamente. Os softwares que existem atualmente possibilitam essa abordagem e podem facilitar a sua compreensão, ou seja, os softwares podem servir como uma espécie de complemento à abordagem experimental. 
A proposição de atividades experimentais mediadas pela tecnologia pode facilitar o entendimento de vários conceitos matemáticos inerentes ao problema proposto. As potencialidades oferecidas pela tecnologia permitem transitar entre os diferentes registros de representação: tabelas, gráficos e analítico. Podemos simular graficamente os dados oriundos da experimentação e obter as equações matemáticas que descrevem o experimento num processo dinâmico.

A utilização dos softwares de geometria dinâmica, por exemplo, possibilita ao aluno criar um ambiente que simule formas geométricas dinâmicas e iterativas, oportunizando ao aluno uma compreensão de diferentes situações problemas e possibilitando um melhor entendimento de conceitos matemáticos. O uso desses softwares pode potencializar o estudo das funções, visto que os mesmos possibilitam uma abordagem dinâmica dos conteúdos.

Segundo Gomez,

$O$ recurso às tecnologias para o ensino e aprendizagem das funções permite uma melhor e mais fácil consolidação do conceito de função em comparação com a abordagem clássica do estudo formal das funções, em que se partia das representações simbólicas e se traduzia por representações tabulares e finalmente por representações gráficas. (GOMEZ, 1997 apud GAFANHOTO e CANAVARRO, 2008, p.5)

O software GeoGebra, por exemplo, é um software gratuito que apresenta várias representações de um objeto matemático, tais como representação gráfica, representação algébrica e representação em tabelas. Deste modo, podemos conectar a utilização deste software com o processo experimental. Com os dados obtidos experimentalmente e plotados no software, o mesmo permite utilizarmos uma ferramenta para a obtenção da função que descreve o comportamento dos dados do experimento realizado. Caso os pontos obtidos no experimento tenham um comportamento linear, podemos encontrar a função que melhor ajuste estes dados por meio da regressão linear. Esta função será apresentada de duas formas: algébrica e gráfica.

O GeoGebra fornece simultaneamente três diferentes visualizações de um objeto matemático: a janela de visualização gráfica, a janela Algébrica e a planilha de Cálculo, possibilitando assim a verificação das representações geométrica e algébrica. Essas representações são adaptadas automaticamente quando modifica qualquer uma delas.

Entretanto, a utilização das ferramentas tecnológicas no ambiente escolar não garante sozinha a melhora na qualidade do ensino, é importante saber como utilizar esses novos recursos em sala de aula, de maneira que possibilite aos alunos um aprendizado mais significativo.

A seguir apresentamos uma sequência didática que busca relacionar o estudo das funções e a Teoria de Representação Semiótica através de atividades experimentais e do uso dos recursos tecnológicos. 


\section{Experimento massa mola}

O experimento intitulado Massa Mola foi aplicado numa turma de primeira fase do curso de Licenciatura em Matemática, do Centro de Ciências Tecnológicas da Universidade do Estado de Santa Catarina-UDESC-CCT. Neste curso está presente a disciplina de Matemática Básica que aborda o nosso objeto de estudo: funções. A escolha também foi motivada pelo fato de ser um curso de Licenciatura, fomentando a formação inicial podemos incentivar a aplicação de metodologias no ensino básico, haja vista que estes alunos serão futuros professores.

A ideia do experimento do sistema massa-mola foi retirada do site Matemática Multimídia, porém a adaptamos para a nossa realidade. Assim, utilizamos materiais disponíveis nos laboratórios de matemática e física da universidade. No experimento, a deformação da mola é dada em função do número de bolinhas de gude que está dentro do pote, conforme a Figura1. Assim, considera-se o número de bolinhas como a variável independente e a deformação da mola como variável dependente.

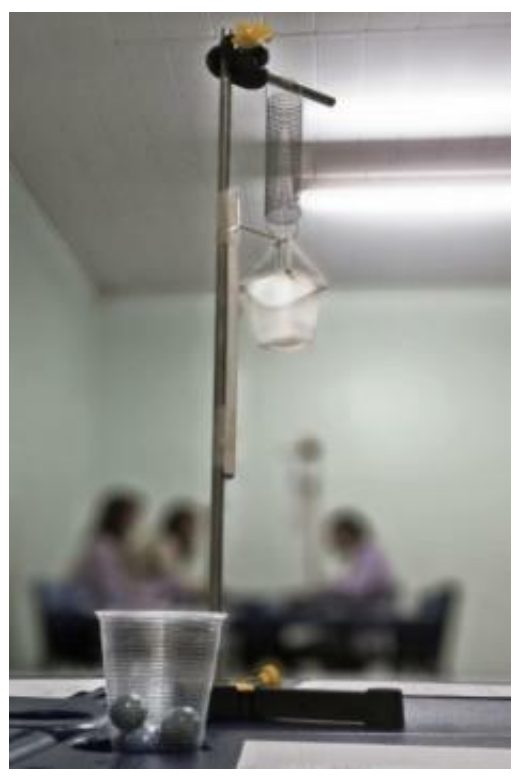

Figura 1: Sistema massa-mola

O principal objetivo da utilização do experimento foi introduzir o conceito de funções por meio da compreensão das grandezas envolvidas e a relação entre elas, em atividades experimentais.

No primeiro momento, os alunos, em grupo, desenvolveram o experimento de acordo com um procedimento que foi fornecido. Neste procedimento, estava descrito quais os passos a serem seguidos. Os alunos deveriam acrescentar uma bolinha de gude no potinho de cada vez, verificar o quanto a mola estava deformando e anotar esses valores numa tabela que também foi fornecida. 
Após a realização destes, eles responderam algumas questões relacionadas com o experimento. Desejava-se que os alunos pudessem:

- Identificar as grandezas envolvidas em cada experimento;

- Identificar a curva que melhor ajuste os pontos obtidos no experimento;

- Obter a razão entre a variação das grandezas envolvidas no experimento;

- Estabelecer a relação algébrica entre as variáveis.

Também foi proposto aos alunos, que após o procedimento realizado e os dados organizados nas tabelas, representar esses dados graficamente no papel milimetrado. Então, a partir dessas informações responderem os questionamentos, que estão no quadro 1:

\section{Quadro 1: Questionário relacionado com o experimento}

a) Quais as grandezas envolvidas no experimento?
b) O conjunto de pontos representados graficamente tem um padrão de comportamento
conhecido? Qual?
c) À medida que acrescentamos as bolinhas, o que acontece com a deformação da mola?
É possível estabelecer uma relação entre as grandezas envolvidas no experimento?
Justifique.
d) Qual é a razão entre a variação das grandezas envolvidas? E o que essa razão
representa no experimento?
e) O que acontece com a deformação da mola quando duplicamos o número de bolinhas?
E quando triplicamos?
f) Existe uma "curva" que melhor descreve o comportamento dos pontos obtidos neste
experimento? Justifique.

\section{Metodologia do experimento}

A metodologia deste experimento consistiu em:

- Orientação aos alunos dos procedimentos e obtenção dos dados;

- Divisão da turma em grupos de 4 alunos, sendo que cada grupo recebeu o instrumento, procedimento e questionamentos referentes ao experimento;

- Obtenção dos dados, utilizando-se o instrumento apresentado na figura 1.

- Organização dos dados em tabela; 
- Construção do gráfico dos dados obtidos na tabela em papel milimetrado e posteriormente num software de geometria dinâmica;

- Resolução das questões propostas (conforme quadro 1), no ambiente lápis e papel;

- Observação, num ambiente computacional, da tendência do tipo de curva determinada pelo conjunto de pares ordenados obtido no experimento.

- Determinação da lei de formação que melhor aproxima o comportamento entre as duas grandezas, por meio do conceito de regressão linear, utilizando as potencialidades do software de geometria dinâmica- GeoGebra.

- Discussão das diferentes formas de representação dos dados obtidos experimentalmente.

\section{Considerações sobre a aplicação}

As considerações são oriundas da análise de algumas observações realizadas em classe, mas principalmente da análise dos protocolos dos grupos entregues após a realização do experimento no ambiente lápis e papel referente aos questionamentos propostos.

Durante a realização do experimento, os grupos, em geral, não apresentaram questionamentos referentes aos procedimentos propostos para a execução da atividade. Também não apresentaram problemas com a utilização e manuseio dos materiais fornecidos para a realização do experimento.

Com os resultados, podemos perceber a facilidade que os grupos apresentaram no processo de conversão do registro de representação em tabelas para gráfica. A representação gráfica pode ser considerada a melhor forma de representação no sentido de analisar o comportamento dos dados, haja vista que o gráfico fornece uma representação visual muito forte. Já na representação em tabelas o comportamento dos dados fica menos evidente e muitas vezes confuso, desta forma percebemos a importância do processo de conversão feito pelos alunos na primeira etapa.

O processo de conversão para a representação algébrica não se deu facilmente, visto que apenas alguns grupos descreveram a relação entre as grandezas utilizando esta representação, sendo um ponto bastante frágil, tendo em vista a importância da representação algébrica. É ela quem modela matematicamente o experimento, fornecendo uma função que descreve e generaliza determinado experimento. A partir desta representação podemos fazer algumas suposições para descobrir outros pontos do experimento. A figura 2, mostra uma resposta do item c (quadro 1), apresentada por um grupo, que encontrou uma representação algébrica para descrever o experimento: 


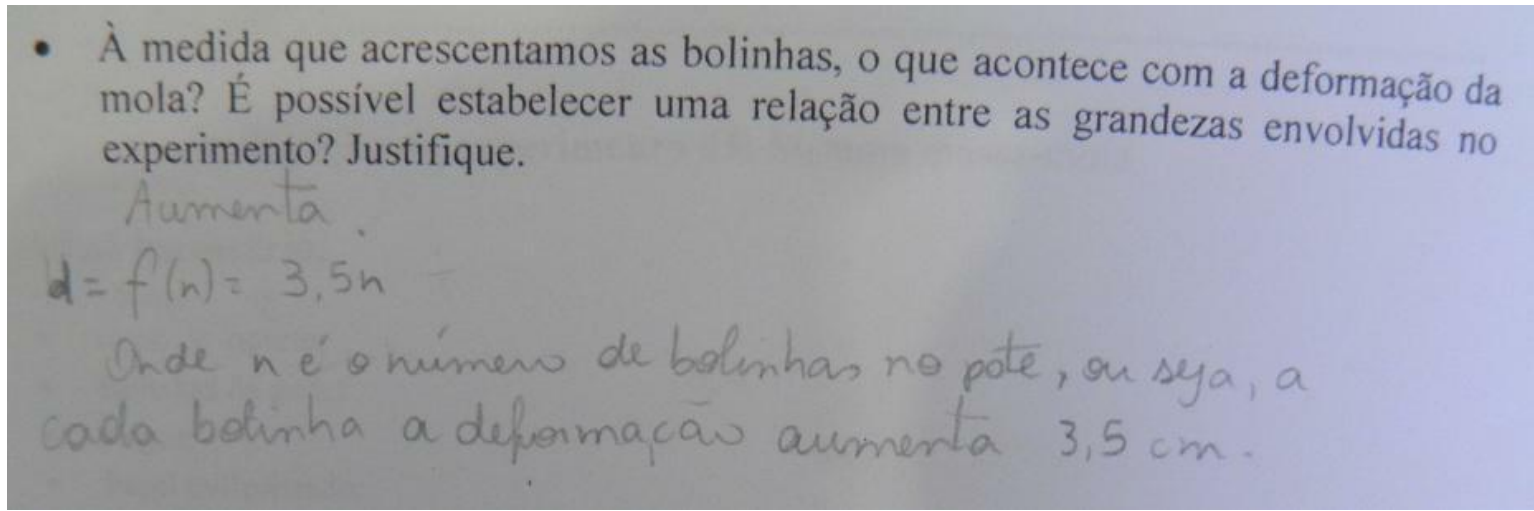

Figura 2: Representação algébrica do experimento

Muitos grupos apresentaram a representação gráfica cujos pontos não estavam alinhados, como podemos verificar na figura 2, assim apresentando maior dificuldade para tentar estabelecer a representação algébrica, haja vista que uma função afim não "passaria" por todos os pontos obtidos no experimento. $O$ fato dos pontos não estarem alinhados é devido aos erros cometidos no momento da realização do experimento, que já eram previstos. Estes erros estão relacionados tanto ao material utilizado quanto pela pouca habilidade do observador.

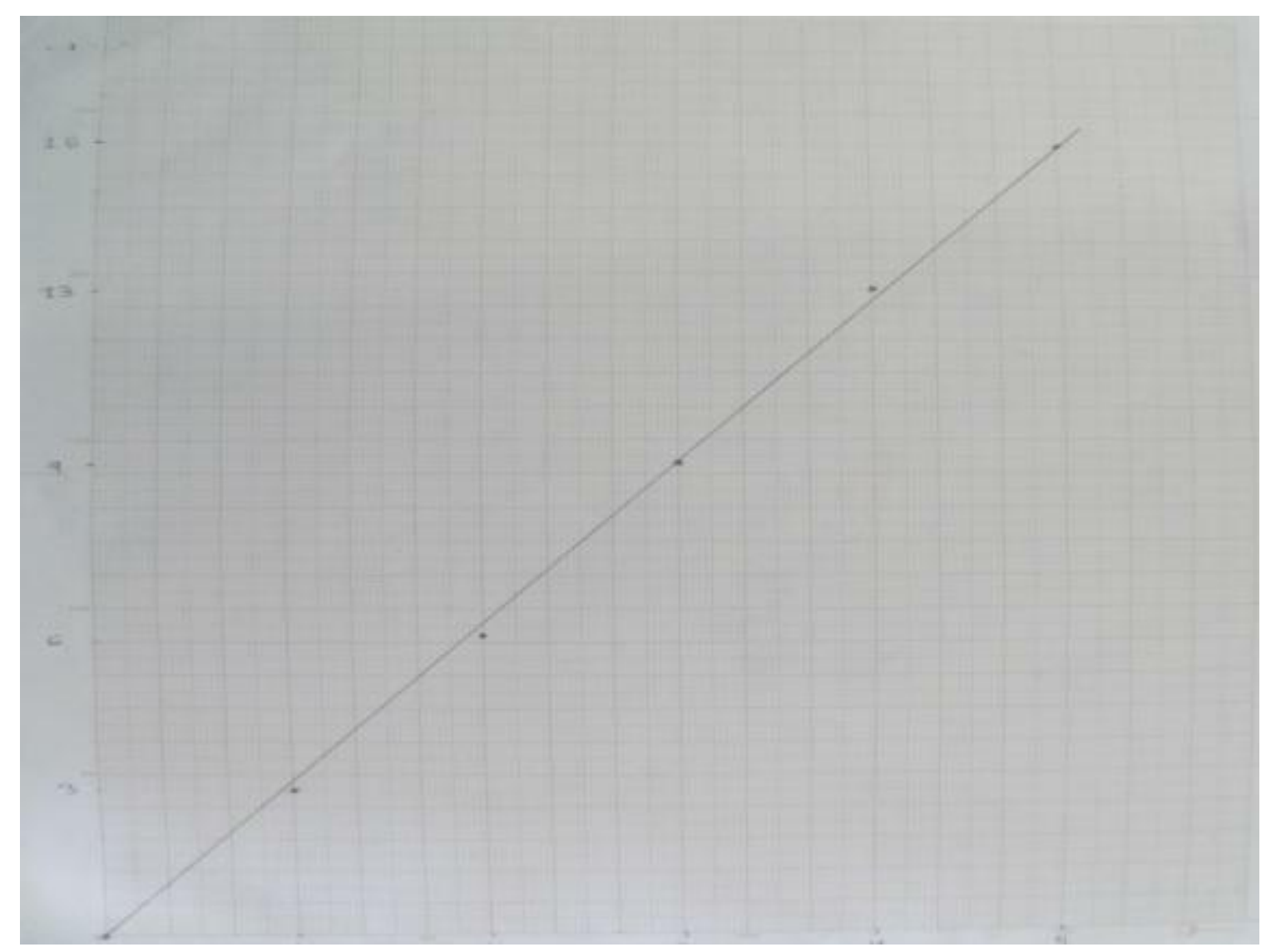

Figura 3: Representação gráfica do experimento

R. B. E. C. T., vol 6, núm. 2, mai-ago.2013 ISSN - 1982-873X 
Em relação à utilização do papel milimetrado notamos que poucos grupos souberam utilizá-lo de maneira correta. Os alunos, em geral, não utilizam este material em suas atividades. Geralmente, nas aulas de matemática os gráficos construídos são apenas esboços, assim a escala não é levada em consideração rigidamente.

Percebemos uma grande dificuldade para os alunos descreverem as grandezas envolvidas nos experimentos. Durante a experimentação este item gerou bastante discussão entre os membros dos grupos, que se questionavam sobre a definição de grandeza. Esta é uma observação bastante interessante, haja vista que para entender o conceito de função é de suma importância que o aluno identifique as grandezas envolvidas. Provavelmente, a ideia de grandeza não é abordada por muitos professores no momento de introduzir o conceito de função e este pode ser um dos fatores que contribuem para que o aluno não consiga resolver um determinado problema. Neste sentido, os alunos também tiveram muita dificuldade de estabelecer a razão entre a variação das grandezas. Os alunos não tinham formalizado os conceitos de razão e de variação. Esses conceitos, muitas vezes, passam despercebidos pelos professores ao trabalharem com as funções, porém são de fundamental importância para construir o conceito da mesma.

Segundo Queiroz et al (2011), a atividade experimental conduz a discussões sobre as possibilidades de registros matemáticos, bem como as nuanças das interpretações da lei deformação, podendo se tornar uma interessante estratégia de discussão, de reconstrução e de prática interdisciplinar no ambiente escolar.

\section{Institucionalização mediada pela tecnologia}

Em um segundo momento, o objetivo da nossa atividade era utilizarmos o ambiente computacional para os grupos identificarem de maneira dinâmica o comportamento dos dados obtidos no experimento.

Utilizando o software GeoGebra, primeiramente foi solicitado aos alunos que eles plotassem os pontos obtidos no primeiro experimento. Então, alguns questionamentos e esclarecimentos foram feitos, em relação às grandezas envolvidas, pontos alinhados, domínio, proporcionalidade, entre outros.

Primeiramente, solicitamos a alguns grupos que fornecessem os dados obtidos durante a atividade experimental e então, confrontamos os dados verificando que nem todos os grupos tinham chego aos mesmos valores, isso devido aos erros de medidas que já haviam sido previstos.

No ambiente computacional, plotamos os dados de um grupo, conforme ilustra a figura 3 , e questionamos os alunos em relação ao modelo matemático que descrevia o fenômeno, mas nenhum aluno soube responder. Verificando os pontos obtidos experimentalmente e representados no gráfico, foram feitos questionamentos aos alunos sobre estes valores obtidos e 
o porquê destes não estarem alinhados. Discutimos também sobre as condições ideais para a realização do experimento, chegando à conclusão de que os pontos não estavam alinhados devido aos erros da massa das bolinhas e algum erro na medição. Entretanto, foi possível observar uma tendência no comportamento dos dados obtidos experimentalmente, concluindo que a curva que melhor descrevia o experimento era uma reta e, portanto era possível obter uma expressão matemática que estabelecesse a relação entre as grandezas envolvidas.

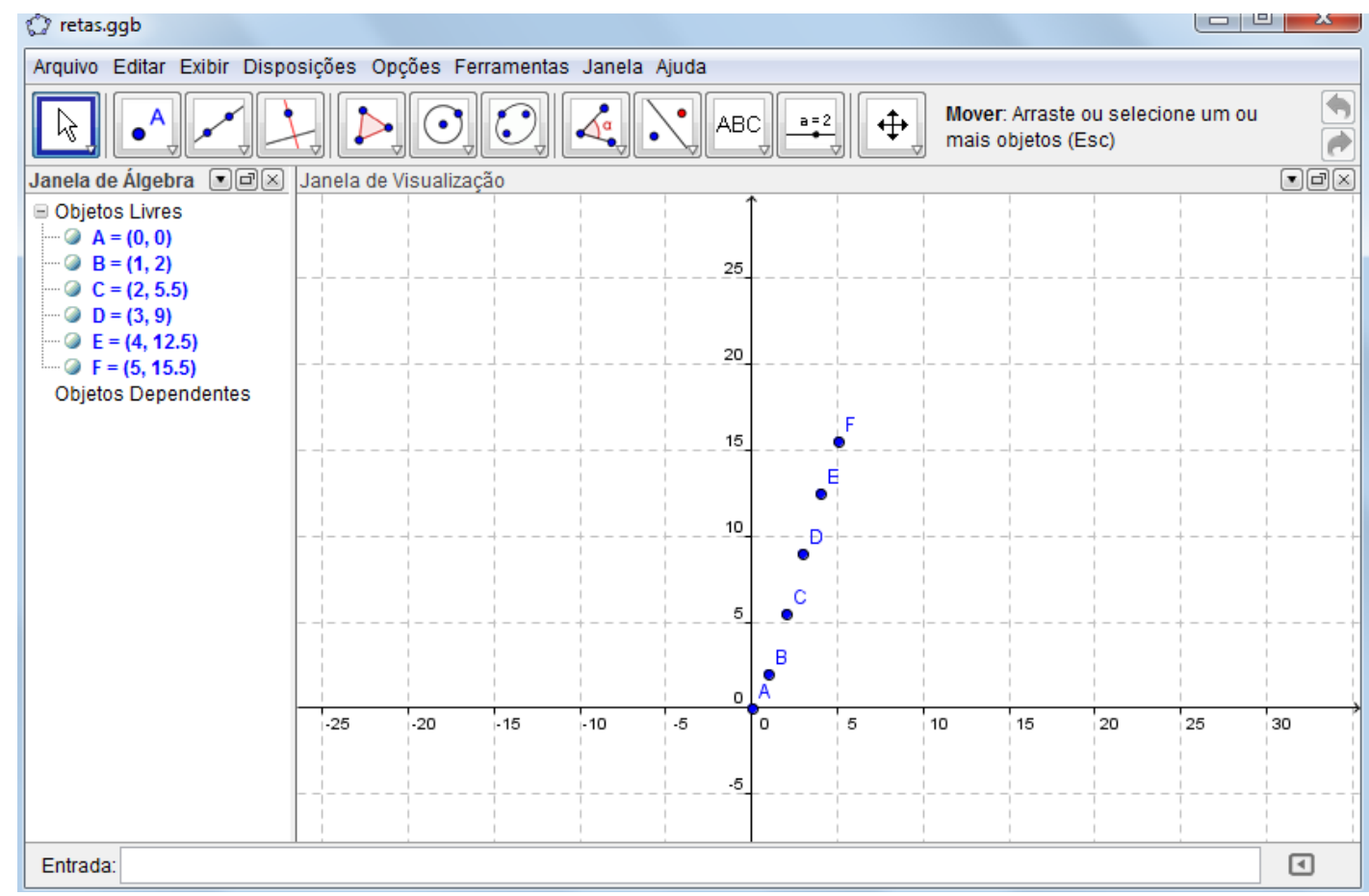

Figura 4: Representação dos pontos obtidos experimentalmente no GeoGebra

Então, realizamos dois procedimentos diferentes para encontrar o modelo matemático: primeiro utilizando o comando de reta definida por dois pontos, selecionando dois pontos quaisquer do experimento, o que nos gerou um reta de equação, onde y representava o valor da deformação da mola em centímetros e x o número de bolinhas.

Aproveitamos o momento para discutir sobre o que representava $\mathrm{o} x$ e $\mathrm{o}$ y no experimento e identificá-los como as grandezas envolvidas. Porém, verificamos junto com os alunos que a reta fornecida não estava de acordo com o processo experimental, visto que, quando não tínhamos bolinhas no copo, a deformação da mola era zero, mas de acordo com a equação fornecida iriamos ter $0,6 \mathrm{~cm}$ de deformação, o que não estava de acordo com o processo experimental.

Desta maneira, concluímos junto com os alunos que era preciso utilizar outro método para encontrar a função que melhor descrevesse aquele experimento. $O$ segundo procedimento 
proposto aos alunos, foi encontrar a reta através do comando da regressão linear, selecionando alguns dos pontos plotados. Então, o software nos forneceu a equação na forma reduzida dada por, , onde y é a o valor da deformação da mola dado em centímetros e x o número de bolinhas. A partir da função obtida observamos junto com os alunos que esta função linear descrevia, em termos matemáticos, o fenômeno representado no experimento. A função encontrada indica uma relação de proporcionalidade direta entre as grandezas envolvidas.

\section{Considerações finais}

O principal objetivo deste trabalho foi desenvolver uma abordagem dinâmica para o estudo de funções, por meio da realização de experimentos práticos e utilização de recursos tecnológicos.

Para atingir nosso objetivo, primeiramente buscamos referenciais teóricos a fim de fomentar nossa pesquisa. Analisamos o desenvolvimento histórico do conceito de função, percebendo que o conceito de função surgiu da necessidade de descrever matematicamente alguns fenômenos naturais. Porém, atualmente, o conceito de função abordado nas aulas de matemática do Ensino Médio, bem como em disciplinas de Matemática Básica e Cálculo Integral e Diferencial do Ensino Superior geralmente valoriza apenas a definição de função através da Teoria de conjuntos. Ocorre que, muitas vezes, os alunos não conseguem compreender o conceito da função, o que reflete nas dificuldades em outras disciplinas, como por exemplo, do Cálculo Integral e Diferencial.

Observamos que a articulação entre os diferentes registros, mediada pelo uso de tecnologias, pode potencializar elementos importantes na aprendizagem de função. Os recursos tecnológicos podem ser muito úteis como instrumento de organização de dados gerados por atividades experimentais e também gerar funções matemáticas que expressam a maneira como os dados obtidos experimentalmente estão relacionados.

Entretanto, é importante ressaltar que nem sempre é possível ou fácil obter uma lei de formação matemática entre duas grandezas, também, que nem todos os conteúdos podem ser potencializados utilizando as atividades experimentais ou recursos tecnológicos. Mas entendemos que ao refletirmos sobre nossa prática, buscando fundamentação tanto teórica quanto prática fica mais simples reconhecer no quotidiano situações que podem ser exploradas no ensino da matemática.

A aplicação desta sequência teve como objetivo principal introduzir o conceito de funções por meio da compreensão das grandezas envolvidas e a relação entre elas, em atividades experimentais. Um diferencial importante desta sequência proposta foi à construção do conhecimento dos alunos. Desta maneira, a definição de função não foi imposta aos alunos, sem 
nenhuma discussão ou contextualização, mas sim, construída por meio de discussão e análise dos dados obtidos, após a realização dos experimentos.

Analisando os resultados da aplicação da sequência didática, percebemos que os alunos foram responsáveis durante a execução da mesma, realizando-a em todos os sentidos, tanto a parte a prática (realização dos procedimentos) quanto a parte dos questionamentos que envolviam o experimento. Essa responsabilidade foi de fundamental importância para o desenvolvimento do trabalho, visto que consideramos os registros para análise. A sequência proposta proporcionou um ambiente de discussão entre os grupos e até mesmo entre a classe no momento da institucionalização do conteúdo em questão.

Salientamos a importância da participação do professor durante esse tipo de atividade, na qual seu papel é de mediador, questionando e instigando o aluno para o mesmo construir conceitos através das discussões.

Percebemos durante o desenvolvimento do trabalho, o quanto esse tipo de abordagem pode proporcionar aos alunos um conhecimento mais significativo. Por meio desse tipo de atividade, é possível motivar os alunos em relação à disciplina e também a determinados conteúdos.

Entendemos que é relevante a realização de uma reflexão sobre a utilização de metodologias diferenciadas para o ensino da matemática, mudando a postura dos professores para que os alunos possam discutir e construir o próprio conhecimento. É importante construir com os alunos uma ideia dinâmica da matemática que está além dos livros didáticos, deixando de lado a ideia da matemática estática, pronta e acabada. 


\section{Referências}

BRASIL. Parâmetros Curriculares Nacionais (Ensino Médio, parte III): Ciência da Natureza, Matemática e suas Tecnologias. Brasília: Secretaria de Educação Média e Tecnológica: MEC/SEMT, 2002.

DUVAL, R. Registres de répresentation sémiotique et fonctionnement cognitif de la pensée. Annales de Didactique et des Sciences Cognitives, IREM de Strarsbourg v.5, p.37-65, 1993 GAFANHOTO, Ana Patrícia e CANAVARRO, Ana Paula. Representações Múltiplas de Funções em Ambiente com Geogebra: um estudo sobre o seu uso por alunos de 9 ano. Projecto Práticas Professional dos Professores de Matemática, 2008. Disponível em: <http://www.ie.ul.pt/pls/portal/docs/1/334274.PDF> Acesso em: 16/04/2012. MACHADO, Silvia Dias Alcântara (Org). Aprendizagem em Matemática: Registros de representação semiótica. Campinas: Papirus, 2003. 1. Ed.

MATEMÁTICA Multimídia. Experimento: Dinamômetro com elástico. Disponível em: < http://m3.ime.unicamp.br/recursos/1006> Acesso em: 08/03/2012.

MORRONE, Wagner; LOZADA, Cláudia de Oliveira, AMARAL, Luiz Henrique e ARAÚJO, Mauro Sérgio Teixeira. Modelagem Matemática e Atividade Experimental como um modelo de integração no ensino de física. Disponível em:

<http://www.sbf1.sbfisica.org.br/eventos/snef/xvii/sys/resumos/T0438-2.pdf> Acesso em: 03/04/2012.

SILVA, Maria Helena Morais e REZENDE, Wanderlei Moura. Análise Histórica do Conceito de Função. / Maria Helena Morais Silva; Wanderlei Moura Rezende. Rio de Janeiro, 1999.

QUEIROZ, Carlos Antônio; RAMOS, Elenita Eliete de Lima. Possibilidades interdisciplinares de Física e Matemática com o uso da prática experimental em Turmas do proeja/cefet-sc. Programa de Especialização em Educação Profissional Técnica Integrada ao Ensino Médio na Modalidade de Jovens e Adultos, do Centro Federal de Educação Tecnológica de Santa Catarina. Florianópolis, SC, 2007. Disponível em: <http://www.ifsc.edu.br/images/stories/file/ PRPPG/monografias /esp_proeja /2007/possibilidades_interdiciplinares_de_fisica_e_matematica_com_o_uso_ da_pratica_experimental_em_turmas_do_proeja_cefet-sc_pdf.pdf> Acesso em: 03/04/2012. NEVES, M. S. Repensando o papel do trabalho experimental, na aprendizagem da física, em sala de aula - um estudo exploratório. Disponível em: <http://www.if.ufrgs.br/public/ensino/vol11/n3/v11_n3_a6.htm> Acesso em: 03/04/2012.

QUEIROZ, Carlos Antônio; RAMOS, Elenita Eliete de Lima e SIPLE, Ivanete Zuchi. Tópicos especiais em ciências I: Representação semióticam tecnologias educacionais e atividades experimentais. Florianópolis: Publicações do IF-SC, 2011. 
REIS, Adinilson Marques. Uma proposta dinâmica para o ensino de função afim a partir de erros dos alunos do primeiro ano do ensino médio. São Paulo, 171 p., 2011. Dissertação (Mestrado em Educação). Pontifícia Universidade Católica de São Paulo. Disponível em:

<http://www.pucsp.br/pos/edmat/mp/ dissertacao/ adinilson_marques_reis.pdf> Acesso em: 02/02/2012.

ZUCHI, Ivanete. A ABORDAGEM DO CONCEITO DE LIMITE VIA SEQUÊNCIA DIDÁTICA: do ambiente lápis papel ao ambiente computacional. Florianópolis, 257 p., 2005. Tese de Doutorado (Doutorado em Engenharia de Produção) - Universidade Federal de Santa Catarina.

Marília Zabel - Universidade do Estado de Santa Catarina - UDESC Joinville - Santa Catarina mari_lia_zabel@hotmail.com Ivanete Zuchi Siple - Universidade do Estado de Santa Catarina - UDESC Centro de Ciências Tecnológicas Departamento de Matemática Joinville - Santa Catarina - ivazuchi@gmail.com 